EPJ Web of Conferences 111,09003 (2016)

DOI: 10.1051/epjconf/201611109003

(C) Owned by the authors, published by EDP Sciences, 2016

\title{
A Covariance Generation Methodology for Fission Product Yields
}

\author{
N. Terranova ${ }^{1,2, a}$, O. Serot ${ }^{2}$, P. Archier ${ }^{2}$, V. Vallet ${ }^{2}$, C. De Saint Jean², and M. Sumini ${ }^{1}$ \\ ${ }^{1}$ Industrial Engineering Department, University of Bologna, Italy \\ ${ }^{2}$ CEA-Cadarache, DEN, DER, SPRC, Saint-Paul-Lez-Durance, F-13108, France
}

\begin{abstract}
Recent safety and economical concerns for modern nuclear reactor applications have fed an outstanding interest in basic nuclear data evaluation improvement and completion. It has been immediately clear that the accuracy of our predictive simulation models was strongly affected by our knowledge on input data. Therefore strong efforts have been made to improve nuclear data and to generate complete and reliable uncertainty information able to yield proper uncertainty propagation on integral reactor parameters. Since in modern nuclear data banks (such as JEFF-3.1.1 and ENDF/BVII.1) no correlations for fission yields are given, in the present work we propose a covariance generation methodology for fission product yields. The main goal is to reproduce the existing European library and to add covariance information to allow proper uncertainty propagation in depletion and decay heat calculations. To do so, we adopted the Generalized Least Square Method (GLSM) implemented in CONRAD (COde for Nuclear Reaction Analysis and Data assimilation), developed at CEA-Cadarache. Theoretical values employed in the Bayesian parameter adjustment are delivered thanks to a convolution of different models, representing several quantities in fission yield calculations: the Brosa fission modes for pre-neutron mass distribution, a simplified Gaussian model for prompt neutron emission probability, the Wahl systematics for charge distribution and the Madland-England model for the isomeric ratio. Some results will be presented for the thermal fission of U-235, $\mathrm{Pu}-239$ and $\mathrm{Pu}-241$.
\end{abstract}

\section{Introduction}

Nuclear data improvement gained an outstanding importance in the scientific community in the last decades, becoming a prior need in advanced nuclear system design. Future innovative nuclear systems are in fact imposing strict safety-by-design standards that require highly accurate engineering parameter uncertainty estimation. It has been quickly recognized that the value and the credibility of any uncertainty analysis was strictly dependent on the scientific quality of variances and correlations $[1,2]$ associated to the basic nuclear data. Supplying complete covariance information gives in fact the opportunity to estimate realistically the uncertainty on integral reactor parameters.

Fission product yields (FY) are fundamental nuclear data for burn-up and activation calculations, including those on decay heat, shielding, dosimetry, fuel handling, waste disposal and safety [3]. Nowadays full uncertainty information are not available in the present data banks (such as JEFF-3.1.1

\footnotetext{
a e-mail: nicholas.terranova@unibo.it
} 
and ENDF/BVII.1) and the nuclear community expressed the need for full fission yield covariance matrices in core and fuel cycle analysis, to take into account complete uncertainty data [4]. Several efforts are being spent to generate reliable FY covariances by different organizations $[7,8]$. In the present work we propose a possible alternative methodology to be applied in FY covariance generation. We chose to adopt the GLSM and the analytical marginalization tools available in CONRAD [5] (COde for Nuclear Reaction Analysis and Data assimilation), developed at CEA-Cadarache. In the following sections we will describe the main features of the procedure we adopted to generate preliminary results on the thermal fission of U-235, Pu-239 and Pu-241. Furthermore, covariance matrices produced through the present methodology have been tested in decay heat uncertainty evaluations using the CYRUS code [6], which allows to estimate decay heat sensitivity coefficients through direct perturbation calculations.

\section{Description of the FY Covariance Generation Methodology}

\subsection{Main Definitions}

Before starting to talk about the procedure that was actually used to generate FY covariances, let us recall some FY definitions which are useful hereinafter. In the ENDF format, two types of FY can be found

- Independent FY, $Y_{\text {post }}(A, Z, M)$, which are the probability to obtain a certain isomer of mass $A$, charge $Z$ and isomeric state $M$ as fission product after the prompt neutron emission, but before any radioactive decay. For this reason we also speak about post-neutron FY.

- Cumulative FY, $C(A, Z, M)$, which take into account radioactive decay. They are in fact the summation of all the contributions to a given isomer overall the entire decay time, since the fission process happened.

In formulas cumulative fission yields are given by

$$
C_{i}=Y_{i}+\sum_{j} C_{j} b(j \rightarrow i),
$$

where we called with $C_{i}$ the cumulative yield for an isomer $i$ identified by the triplet $(A, Z, M)_{i}$, with $Y_{i}$ its corresponding independent yield $Y_{i}$ and with $b(j \rightarrow i)$ the branching ratio which gives the probability that an isomer $j$, characterized by $(A, Z, M)_{j}$, decays into $i$.

Summing over the charge and the isomeric state we should obtain what is usually called independent mass yield, $Y(A)=\sum_{Z, M} Y_{\text {post }}(A, Z, M)$. Independent mass yields $Y(A)$ differ of few percent by chain yields, $C h(A)$. The latters are equal to the sum of all stable long-lived cumulative yields for a given mass chain [3]. The main reason is that unstable neutron-rich fission isomers mostly decay through $\beta$-particle emission, without any mass change. In the following sections the semi-empirical models we used to reproduce the JEFF-3.1.1 library and to generate covariances will be discussed.

\subsection{FY Semi-Empirical Models}

In the present FY nuclear data library, such as JEFF-3.1.1, semi-empirical models are employed to calculate independent fission yields. Such models are used in a fitting procedure to interpolateextrapolate missing data, especially for those fissioning systems which have few available experimental data. Independent fission yields can be written as the product of three factors

$$
Y_{\text {post }}(A, Z, M)=Y_{\text {post }}(A) f(A, Z) R(A, Z, M)
$$


where $f(A, Z)$ is the charge distribution giving the fractional independent yields and $R(A, Z, M)$ is the isomeric ratio. In JEFF-3.1.1 evaluation mass yields interpolation and extrapolation are deduced performing a multi-Gaussian systematics calculation to fit chain yields [3, 9]. Multiple Gaussians can in fact well represent the general trends of mass distributions, if the fluctuations due to prompt neutrons emission are ignored. Such systematics can be roughly justified thanks to the multi-channel fission theory proposed by Brosa [10]. In the Brosa model, however, only pre-neutron mass yields distributions can be represented by a summation of five Gaussian corresponding to three fission modes. The Standard I and Standard II modes are responsible for the asymmetric and the very asymmetric fission. The symmetric Super Long instead is centered in $A_{f} / 2$, where $A_{f}$ is the mass of the compound nucleus. In formulas we have

$$
Y_{p r e}(A)=\sum_{i=s 1, s 2} \sum_{\Delta= \pm D_{i}} \frac{N_{i}}{\sqrt{2 \pi} \sigma_{i}} \cdot e^{-\frac{\left(A-A_{f} / 2-\Delta\right)^{2}}{2 \sigma_{i}^{2}}}+\frac{2 N_{s l}}{\sqrt{2 \pi} \sigma_{s l}} \cdot e^{-\frac{\left(A-A_{f} / 2\right)^{2}}{2 \sigma_{s l}^{2}}}, \text { with } \sum_{i=s 1, s 2, s l} N_{i}=1.0 .
$$

$N_{i}$ are the weights of the several fission modes, $\sigma_{i}$ the corresponding widths and $D_{i}$ the deviations from the mass symmetry for the two asymmetric fission modes.

In JEFF-3.1.1 FY charge distributions $f(A, Z)$ are obtained using Wahl systematics for independent fission yields [11] and the Madland England [12] model is used instead to retrieve isomeric ratios.

In the present work, to represent JEFF-3.1.1 in a physical consistent way, we decided to adopt the Brosa model to represent pre-neutron mass distributions as it was meant to be. In order to finally obtain the independent mass yield distribution we seek, a prompt neutron emission probability model based on Gaussians distributions has been used. The Wahl systematics has been applied directly to the pre-neutron distributions, which have to be multiplied by prompt neutron emission probabilities $p_{A}(v)$ to give post-neutron yields. Such functions have been assumed as Gaussians centered in $\bar{v}$. The average number of prompt neutrons emitted $\bar{v}$ is a function of the pre-neutron mass $A_{\text {pre }}$ of the primary fission fragments. Experimental measurements have shown a saw-tooth distribution for $\bar{v}\left(A_{\text {pre }}\right)$, and such shape is roughly maintained between different fissioning systems. In formulas we get then

$$
Y_{p o s t}(A, Z, M)=\left[\sum_{v_{i}=0}^{\infty} Y_{p r e}\left(A+v_{i}\right) \cdot p_{A+v_{i}}\left(v_{i}\right) \cdot f\left(A+v_{i}, Z\right)\right] \cdot R(A, Z, M) .
$$

Further explanation on the prompt neutron emission model can be found in [13]. Cumulative FY can be subsequently obtained through the $\mathbf{Q}$-matrix formalism [3].

\subsection{GLSM and Marginalization}

The FY models presented in Sect. 2.2 have been employed in a GLSM to retrieve adjusted model parameters able to represent JEFF-3.1.1 FY library. Using CONRAD, we minimize a cost function [14]

$$
\chi^{2}(\vec{x})=\left(\vec{x}-\vec{x}_{\text {prior }}\right)^{T} \mathbf{M}_{\text {prior }}^{-1}\left(\vec{x}-\vec{x}_{\text {prior }}\right)+[\vec{y}-\vec{f}(\vec{x})]^{T} \mathbf{M}_{y}^{-1}[\vec{y}-\vec{f}(\vec{x})]
$$

using an iterative Newton-Raphson algorithm which gives

$$
\begin{gathered}
\mathbf{M}_{x}^{(n-1)}=\left[\mathbf{M}_{\text {prior }}^{-1}+\mathbf{G}^{(n-1)^{T}} \mathbf{M}_{y}^{-1} \mathbf{G}^{(n-1)}\right]^{-1}, \\
\vec{x}^{(n)}=\vec{x}^{(n-1)}-\mathbf{M}_{x}^{(n-1)} \cdot\left[\mathbf{G}^{(n-1)^{T}} \mathbf{M}_{y}^{-1} \cdot\left(\vec{f}\left(\vec{x}^{(n-1)}\right)-\vec{y}\right)+\mathbf{M}_{\text {prior }}^{-1}\left(\vec{x}^{(n-1)}-\vec{x}_{\text {prior }}\right)\right] .
\end{gathered}
$$

Without entering into the details on how Eq. 6 and Eq. 7 are derived (see Ref. [15]), posterior model parameters $\vec{x}$ and their covariance matrix $\mathbf{M}_{x}$ can be found once priors $\vec{x}_{p r i o r}$ and new experimental 
values $\vec{y}$ are available, provided with corresponding covariance matrices $\mathbf{M}_{\text {prior }}$ and $\mathbf{M}_{y} \cdot \vec{f}(\vec{x})$ are the FY models just presented and $\mathbf{G}^{(n)}$ their derivatives. We performed the adjustment using JEFF-3.1.1 evaluated FY data as pseudo-experimental values. Brosa fission mode characteristics, $\bar{v}$ values, a single width for all the prompt neutron Gaussian probabilities, Wahl model parameters and the Madland England cut-off were simultaneously adjusted to achieve a satisfactory JEFF-3.1.1 representation. Such procedure was performed including extra experimental values concerning miscellaneous fission quantities, as the total prompt neutron emission probability distribution and the total average number of prompt neutrons emitted by the fissioning system. The parameter covariance matrix $\mathbf{M}_{x}$ was finally deduced enabling a retroactive analysis fitting only Brosa, Wahl and Madland England parameters.

To better reproduce also the uncertainties provided by the European library, an analytical Marginalization technique [16] has been set up to introduce systematic uncertainties on the normalization of FY charge distribution. The main issue of this phase was to represent all the uncertainties of the hundreds of independent FY available for each fissioning system. For U-235 for example, we focused on the normalization of Xe and Cs distributions. Acceptable results have been obtained also for other fission products, as demonstrated by the decay heat uncertainty calculations.

\section{Preliminary Results}

In this section some results achieved using the methodology just described are presented. In particular we will show the capabilities of this FY model to represent JEFF-3.1.1 evaluated data, with the main goal to obtain consistent correlation matrices. Some results on the decay heat uncertainties calculation using the CYRUS code will be included.

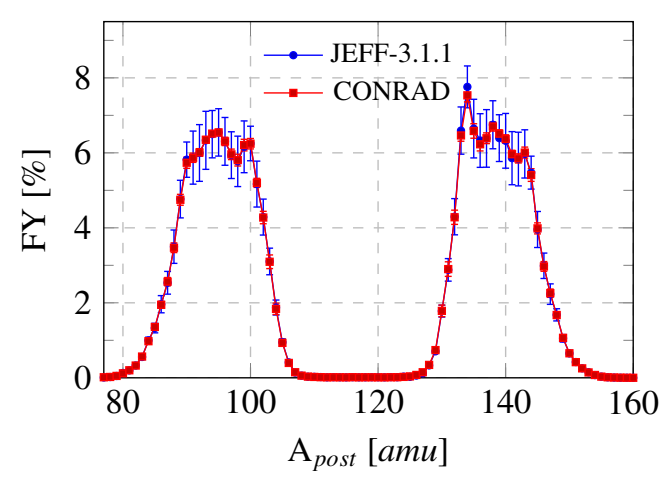

(a) Mass Independent FY Distribution U-235

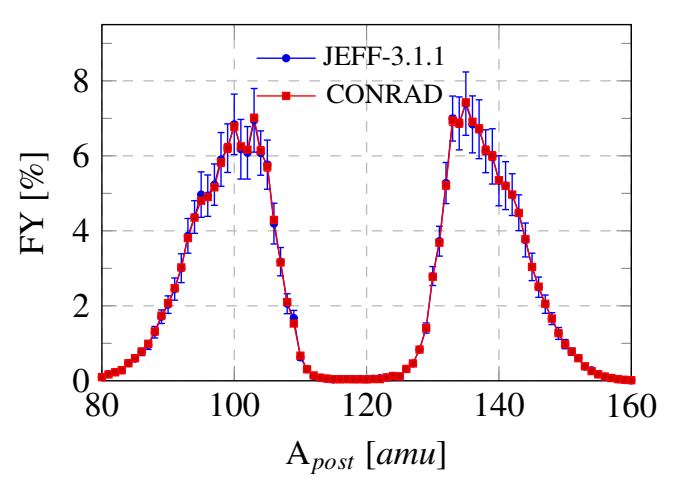

(b) Mass Independent FY Distribution Pu-239

Figure 1: Comparison between JEFF-3.1.1 and CONRAD independent mass yields distribution for the thermal fissions of U-235 (left) and Pu-239 (right).

\subsection{Covariance Matrix Generation}

Satisfactory comparisons between CONRAD and FY evaluated in JEFF-3.1.1 have been performed for three thermal fissioning systems: U-235, Pu-239 and Pu-241. Hereinafter a few results will be presented just to point out the potential of the present methodology, without pretending to be exhaustive. Fig. 1(a) and Fig. 1(b) show how present FY models can nicely represent JEFF-3.1.1 mass independent fission yields for the thermal fission of U-235 and Pu-239. Similar results can be obtained also for the thermal fission of Pu-241. FY adjustment has been performed including miscellaneous 


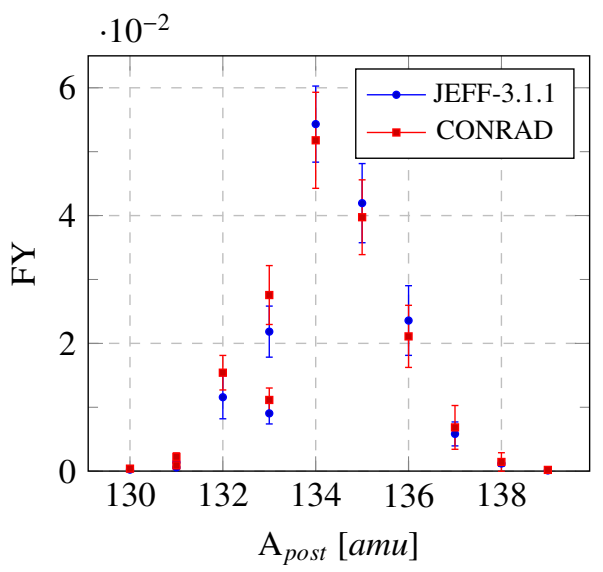

(a) Tellurium FY for Pu-241 fission

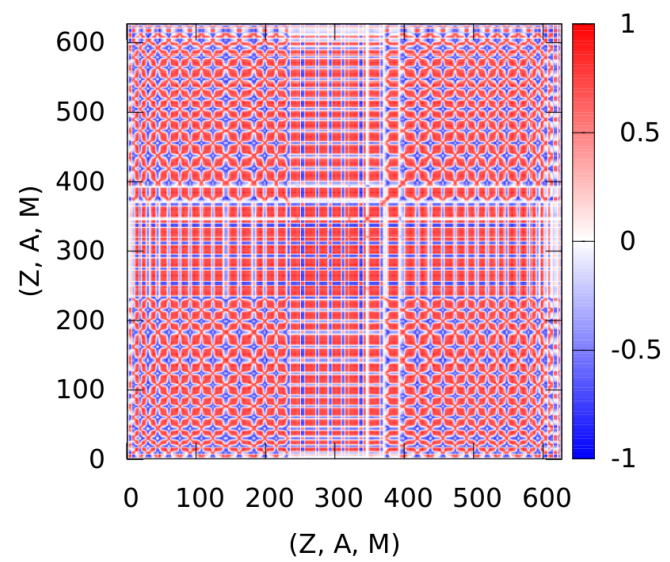

(b) Pu-241 Correlation Matrix

Figure 2: Pu-241 isomeric independent fission yields distribution (left, only Tellurium has been shown) and correlation matrix (right). Only FY $>10^{-7}$ have been considered. Matrix coordinates are just cardinal numbers for a list of $\sim 600$ fission products, ordered for ascending charge, mass and isomeric state.

fission quantities such as the total prompt neutron emission probability $P(v)$ and the average number of prompt neutrons emitted by the fissioning system $\bar{v}_{P}$, which have been effectively reproduced [13]. In Fig. 2(a) and 2(b) Tellurium isotopic distribution and the full independent correlation matrix are presented for the thermal fission of Pu-241. Even isomeric fission yields are well described by the models and a correlation matrix can be found preserving agreement between CONRAD and JEFF results.

\subsection{Decay Heat Uncertainty Estimation}

To test fission yield covariance matrices, we used the CYRUS code, which performs decay heat uncertainty estimations. For the elementary thermal fission of U-235, the decay heat uncertainties due to U-235 fission yields provided by JEFF-3.1.1 and by CONRAD have been compared (see Fig. 3(a)). Results show reasonable agreement between the propagation of JEFF-3.1.1 and CONRAD uncertainties, without including correlations between data. Full CONRAD FY matrices propagation on the other hand provoked a dramatic decay heat uncertainty reduction, which has to be further investigated. The same exercise was repeated even for a PWR-UOX pin-cell (Fig. 3(b)), providing same conclusions and showing that FY uncertainties for fission products which are sensitive to such problems are well represented by the present methodology.

\section{Conclusions}

A methodology to generate FY covariance matrices has been presented. The main goal of such procedure is to reproduce the existing European FY library JEFF-3.1.1 in a physical consistent way and to generate covariances. Such covariances have been tested for applications in decay heat uncertainty calculations, showing how FY correlations can have an important impact on integral reactor parameter uncertainty reduction. 


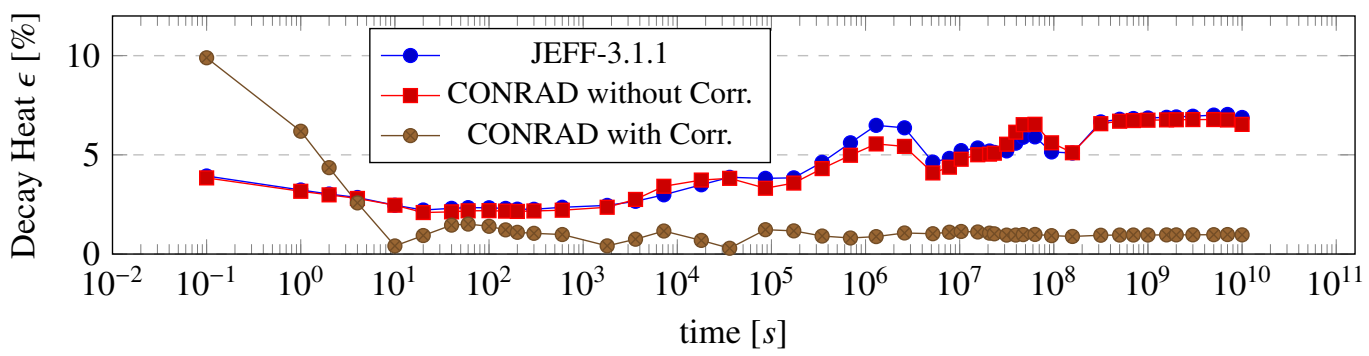

(a) Elementary Fission

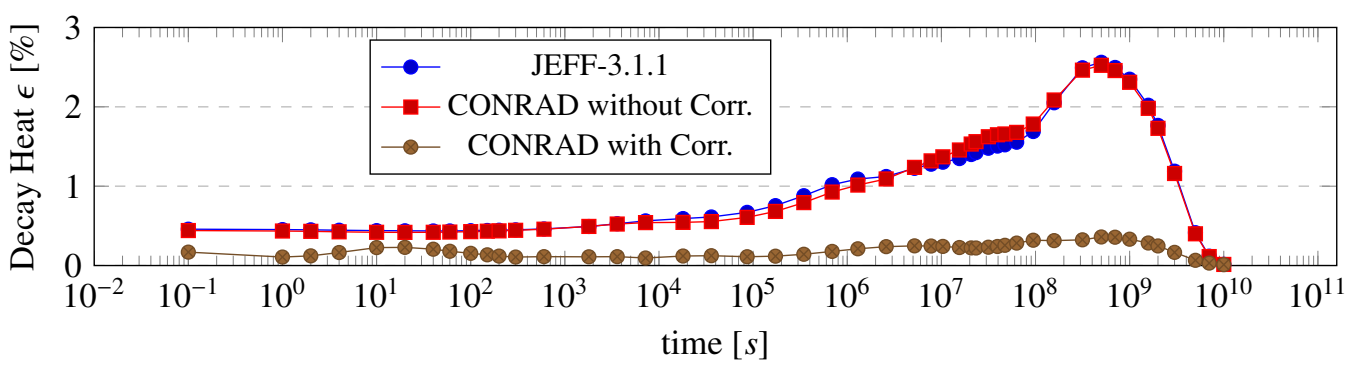

(b) PWR-UOX Pin-Cell

Figure 3: Decay heat uncertainty estimation due to U-235 FY propagation in elementary fission (top) and PWR-UOX pin-cell (bottom) calculations.

\section{References}

[1] G. Palmiotti et al., Nuclear Data Sheets 118, 596 (2014).

[2] G. Aliberti et al., Annals of Nuclear Energy 33, 700 (2006).

[3] R. Mills, "Fission Product Yields Evaluation", PhD Thesis, University of Birmingham, United Kingdom (1995).

[4] R. Mills, WPEC SG-37 Proposal, (2012).

[5] P. Archier et al., Nuclear Data Sheets 118, 488-490 (2014).

[6] V. Vallet et al., PHYSOR-2014, Kyoto (2014).

[7] L. Fiorito et al., Annals of Nuclear Energy 69, 331, (2014).

[8] M. T. Pigni et al., Nuclear Data Sheets 123, 231-236, (2015).

[9] M. A. Kellett et al., OECD-NEA JEFF Report 20, (2009).

[10] U. Brosa et al., Physics Reports 4, 167 (1990).

[11] A. C. Wahl, "Systematics of Fission-Product Yields", LA-13928, (2002).

[12] D. G. Madland, T. R. England, Nuclear Science and Engineering 64, 859 (1977).

[13] N. Terranova et al., Nuclear Data Sheets 123, 225-230 (2015).

[14] C. De Saint Jean et al., Journal Korean Physical Society 59, 1276 (2011).

[15] D. Smith, Probability, Statistics and Data Uncertainties in Nuclear Science and Technology, OECD-NEA Committee Series, (1990).

[16] B. Habert et al., Nuclear Science and Engineering 166, 276 (2010). 